\title{
Queueing Theory Based Minimum Spanning Tree for Energy Consumption in Wireless Network
}

\author{
S. Nazreen Begum ${ }^{1}$, Dr. S. Bharathidass ${ }^{2}$ \\ ${ }^{1}$ Research Scholar (Part Time), Dept of Statistics, Periyar EVR College, Tirchirappalli, India \\ ${ }^{2}$ Assistant Professor, Dept of Statistics, Periyar EVR College, Tirchirappalli, India
}

\begin{abstract}
Wireless networks consist of number of sensor nodes which are equipped with data transceivers that enable them to communicate with each other to perform collaborative sensing tasks over a given area. Data collection is one of the important factors that affect data transmission and processing in wireless sensor networks. This paper discourses this issue by resources of the paths delay analysis. Queueing theory for wireless networks are of various kinds that cater to various different needs of researchers and scientists. Since each of these have a unique set of advantages and disadvantages, it becomes necessary for us to understand which of these might suit a particular scenario best. Queueing theory model the connectivity time packet sending time it could be loss of the data transmission. Our proposed model use M/M/1/N type queueing network model with holding nodes for wireless network and strategy approximate iterative algorithms. Experimental consequences show that the model is reliable with the real data. Queueing model leads to high energy consumption on their network. Our proposed method has to implement this problem and use efficient data collection using queueing model on the network. Also using the Minimum Spanning Tree (MST) its more than one geometry based on the link configuration. There are two channels like vertical channel Take a different parameter to show the result like as throughput, delivery ratio, delay, energy consumption, and then data collection efficient on networks.
\end{abstract}

Keywords: Queueing theory, Minimum Spanning Tree, Energy consumption, Data collection, Wireless network

\section{Introduction}

We consider that energy corresponds to the length of the waiting queue in the established limited capacity of the queueing theory network perfect. When the distance of the waiting queue reaches the maximum, the node is congested in the queueing network model. Therefore, a typical WSN is exhibited by $\mathrm{M} / \mathrm{M} / 1 / \mathrm{N}$ type queueing network model. The method of modeling based on the topology of nodes in WSNs and performance analysis of the data collection capacity have been proposed. According to the topology of WNs and working characteristics, appearance, transporting and leaving relations of transmission nodes, border nodes and sink node are examined, and data flow balance reckonings are obtained. In order to assess the congestion situation in the queueing network, and get real effective arrival rates and broadcast rates in the model, holding nodes were added in the queueing network model and corresponding queueing network perfect is expanded. By examining the queueing model with blocking prospect, to obtain the performance index of the arrangement when it is in steady state, estimated iterative algorithms are designed. The performance parameters of nodes perfect in the WSNs are intended using limited repetition times. The optimal values for packets buffer sizes locations are obtained for broadcast nodes, boundary nodes and sink nodes.

In a typical high-throughput queuing theory, nodes periodically send probe to their neighbors to amount the quality of their next to links. During direction discovery, a node estimations the cost of the path by linking its own measured metric of together links with the path cost accumulated on the route discovery packet. The minimum spanning path with the finest metric is then designated. High-throughput protocols require the nodes to cooperate in order to derive the path metric, thus relying on the supposition that nodes behave properly during metric computation and propagation. However, this supposition is difficult to guarantee in wireless networks that are vulnerable to attacks coming from both insiders and outsiders, due to the open and shared nature of the medium and the Multihop characteristic of the communication.

The significant measures of a sensor node is its energy supply. In fact, the excellence of a wireless network is measure by its coverage, and thus the energy independence of each constituent node is of utmost importance. In calculation, due to the node reduced extents and, in most cases, inaccessibility in the places they are deployed, it is important to optimize energy supply wherever possible. In fact the nearest source of energy is the node surrounding environment. Energy reaper is the part of some nodes responsible to do the best job of collecting, conditioning, and storing energy from the surrounding energy source.

An aggressive path selection introduces new vulnerabilities and provides the attacker with an increased arsenal of attacks leading to unexpected consequences. They are secondhand for maintaining itself and the rest of the sensor node mechanisms operating over the lengthiest possible periods of time. The oldest liveliness harvesters were purely motorized: windmills and water wheels are two of the classical examples. More recent mechanical harvesters have been used in the wrist watches for quite some time.

The priorities and blocking mechanisms with open-loop queueing network performance analysis, and queueing network parameters on the approximation and error estimates. Using queueing network delay evaluation, problems of analyzing modeling and path planning in various fields are successfully resolved. However, the topology of the wireless network is very complex requiring a large number of transmission paths within the network. Thus, path delays are most computational overhead and slow 


\section{International Journal of Science and Research (IJSR) \\ ISSN (Online): 2319-7064}

Index Copernicus Value (2013): 6.14 | Impact Factor (2015): 6.391

down the speed. Therefore, performance evaluation using queueing network method for delay optimization is rarely mentioned in the field of wireless network.

\section{Related Work}

In the works, a number of investigation results on queuing presentation analysis for wireless sensor networks have been reported. A typical method in queuing analysis for wireless sensor networks is showing a sensor node as a finite FIFO queue using a incessant time Markov chain assuming sensor nodes alternate between active and sleep modes. The interchange of active/sleep mode may help reduce the energy consumption of a sensor node. However, the sleep mode will cause extra delay and packet loss.

The effectiveness of cluster-based distributed sensor networks depends to a large extent on the coverage provided by the sensor deployment. A virtual force algorithm as a sensor deployment strategy to enhance the coverage after an initial random placement of sensors, for a given number of sensors, the virtual force algorithm attempts to maximize the sensor field coverage. A combination of attractive and repulsive forces is used to determine virtual motion paths and the rate of movement for the randomly-placed sensors. Once the effective sensor positions are identified, a one-time movement with energy consideration incorporated is carried out, i.e., the sensors are redeployed to these positions.

Regardless of the tests mentioned above, there must be trade-off between energy consumption and resource operation. However, service time for each arrival packets increases as the size expands, therefore, number of packets waiting in queue in the energy consumption. The loss of some vid-eo and the packets might not extremely affect overall data in destination, so that outstanding packet can convey the compulsory message to the listener. In the case of loss of image packet, it is problematic to rearrange whole surround in order to get innovative picture back. In this paper we presented quality of service quantity by analyzing the packets in the queue by using Queueing theory.

Based on the channel model, the waveguide technique for communication is developed to address the high attenuation challenges of waves through soil. Carefully scheming the waveguide parameters, the path loss can be greatly reduced. The relay coils constituting the queueing theory do not consume any energy and the cost is very small. The queueing theory is not a continuous structure of a real waveguide hence it is relatively flexible and easy to deploy and maintain. When the time of individual node communication is not normal or if there is energy depletion, the packets to be processed are queued in the blocking node. If the blocking cannot be solved in time, blocking occurs in all the nodes of the link path, resulting in the congestion of the entire network. Then, a spare second best path except the optimal path becomes necessary to ensure that the entire
WSN operation is efficient. This requires that to keeping the high efficiency of the path selection in a system for communication, and the searching paths occupy the smallest delay.

\section{Proposed System}

In our proposed model have using a multicast routing based queueing model in network. A Low Propagation Delay Multi Cast Routing based queueing theory forms a direction from source to the destination which comprises of $n$ numbers of multi-sub paths during the transmitting path construction. Multi sub pathways are helpful for sub paths procedure sender to its two-hop neighbors thru a communicate node in the neighborhood of both sender and receiver nodes. Basically this method is useful to keep data collision at receivers since they receive packets from different relay nodes. Wireless sensors have more traffic and not secure data transmission on the network. They use three stages sleep mode, awake mode, idle mode. To find out the destination place use shortest route on network. We have proposed a minimum spanning tree algorithm in wireless sensor networks to allow each node to select its next hop with the highest successful delivery rate of the minimum energy consumption and increasing a throughput reduce the delay on network.

The queueing theory solves the troubles of customary technique in FIFO model in network. On the other hand, deploy data collection to attach the wireless nodes in such network is demanding due to the far above the ground operation cost, the multifaceted shape of the communication variety of the data collection and the important impacts of the swelling breakdown and relay loop displacement. To appointment the use problems in the queueing model network have not be addressed.

\subsection{Node Energy Savings based on Queueing model:}

We next evaluate the energy saved by the proposed queueing model approach. Assume the sensor node has three basic energy consumption types sensing, transmitting and receiving, and these power values (energy per unit time) are $E_{s}, E_{t}$ and $E_{r}$, respectively. If we select all sensors that reported the target for querying, the total energy consumed for the event happening at time instant $t$ can be evaluated using the following equation:

$$
\begin{aligned}
& E_{1}(t)=N_{r e p}(t)\left(E_{t}+E_{n}\right) T_{1} \\
& E_{2}(t)=N_{n} T_{n} \\
& E=\sum_{N_{n-\text { start }}}^{N_{n-\text { gnd }}} E(t)
\end{aligned}
$$

$$
\Delta E=E-E^{*}=S \sum_{t_{t-\text { start }}}^{t_{\text {end }}}(\text { total energy }(t)-\text { consumed energ }(t))
$$

Volume 5 Issue 6, June 2016 www.ijsr.net 


\section{International Journal of Science and Research (IJSR) \\ ISSN (Online): 2319-7064}

Index Copernicus Value (2013): 6.14 | Impact Factor (2015): 6.391

Where $E_{1}$ is the energy required for queueing model reporting the detection of an object the parameters $T_{1}$, denote the lengths of time involved in the transmission and reception, which are directly proportional to the sizes of data, control messages to query sensors, and the detailed sensor data transmitted to the cluster head. The parameter $T_{s}$ is the time of sensing activity of sensors. The parameters $E$ denotes the total energy in this case for target localization from $t_{\text {start }}$ to $t_{\text {end }}$. Similarly, for the proposed probabilistic localization approach, we have

Where

$C=E_{r} T_{1}+\left(E_{t}+E_{r}\right) E_{\text {no.of node energy }}$

Since $N_{n}(t)$ is always less than or equal to $D_{n}(t)$,

We have

$\nabla \mathrm{E} \geq 0$

So we consumed energy and rectifying the damaged node I during data transmission using queueing model in FIFO network.

The optimal queueing operation strategies are investigated in both single and two dimensional queueing networks where the nodes are dispersed also arbitrarily or according to a usual lattice. Validated by together hypothetical inference and simulations, the deployment strategy can build a reliable queueing theory system that is healthy to lump failure and communicate coil dislocation with smallest amount cost.

\subsection{FIFO based MST in queueing theory}

This algorithm is known as Low Propagation multi path routing based data collection in the network to analysis queueing model a low propagation delay minimum spanning tree a route from source to the destination which contains of $\mathrm{n}$ amounts of multi-sub paths during the routing path construction. Multi sub paths are helpful for sub paths to form sender to its two-hop neighbors through a relay node in the neighborhood of both sender and receiver nodes.

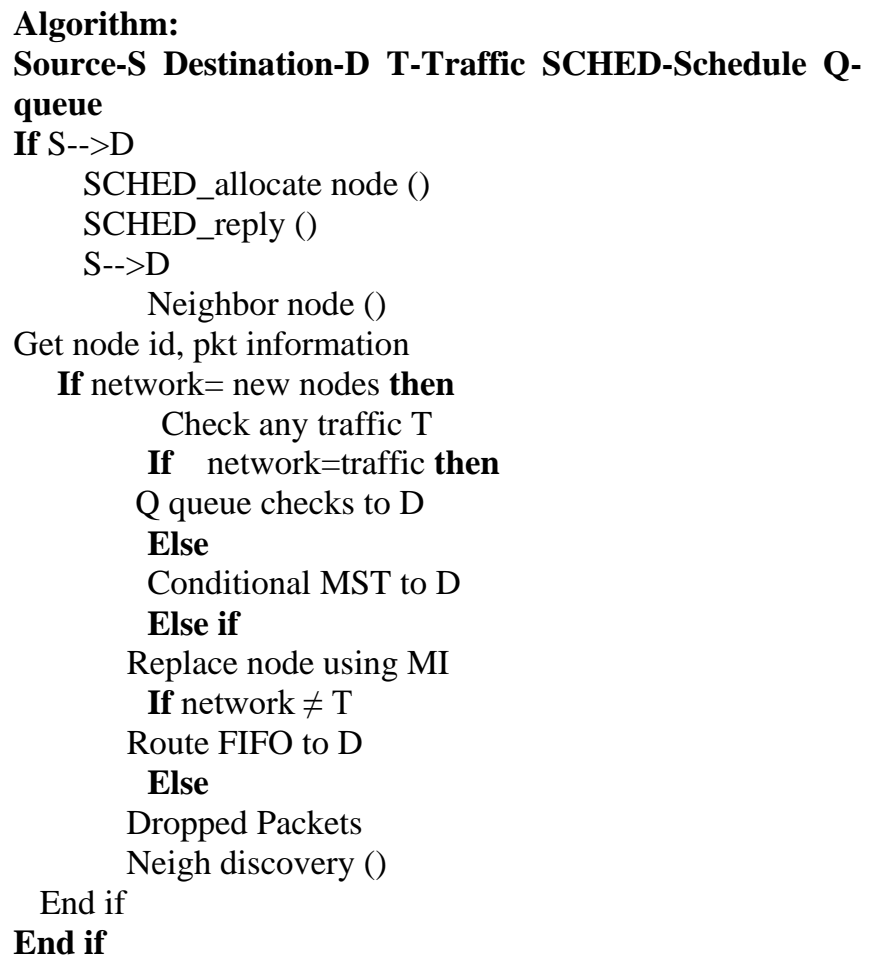

Traffic model based on the sensor network, in the queueing model network, have any interrupt or waiting on the transmission is traffic on the network. But in wireless network data transferring could create big interrupt at the time. So the data communications are loss and more traffic on the network. This term refers to like more than one way for communication signal that generates that is referred to network signal model. The more than one geometry grounded on the link conformation. In this term have hopped by hop communication model on their network.

\section{Performance Analysis}

The Purpose of our reproduction to analyze the presentation of the AODV by using interlocks Networks. The repetition surroundings are shaped in NS-2, in that provides preserve for a wireless networks. NS-2 was using C++ language and it has used for an Object Oriented Tool Command Language. It came as an extension of Tool Command Language (TCL). The execution were approved out using a wireless environment of 100 wireless nodes rootless over a simulation area of 1200 meters x 1200 meters level gap in service for 10 seconds of reproduction time. The network grounded data processing or most exclusive and data announcement level on their performance on the network. Hence, the simulation experiments do not account for the overhead produced when a multicast members leaves a group. Multiple sources create and end sending packets; each data has a steady size of 512 bytes. Each mobile node to move randomly on their network, it's more and most expectable on their networks.

\begin{tabular}{|c|c|}
\hline Parameters & Value \\
\hline version & Ns-all-in-one 2.28 \\
\hline Protocols & AODV \\
\hline Area & $1200 \mathrm{~m} \times 1200 \mathrm{~m}$ \\
\hline Broadcast Area & $250 \mathrm{~m}$ \\
\hline Transfer model & UDP, CBR \\
\hline Data size & 512 bytes \\
\hline
\end{tabular}

\subsection{Throughput Performance}

The ratio of throughput concert overall network performance improve network performance and packet delivery ratio and decrease packet delay.

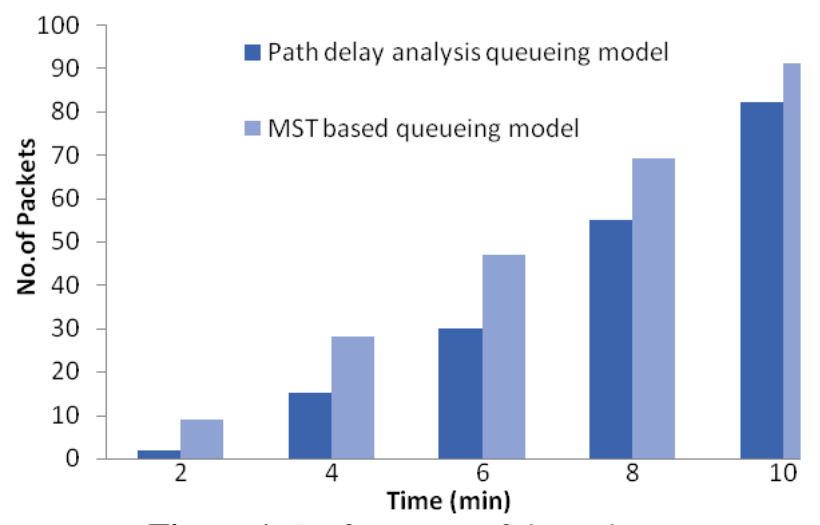

Figure 1: Performance of throughput

Volume 5 Issue 6, June 2016 www.ijsr.net 


\section{International Journal of Science and Research (IJSR) \\ ISSN (Online): 2319-7064}

Index Copernicus Value (2013): 6.14 | Impact Factor (2015): 6.391

\subsection{Delivery ratio performance}

The packets are delivered since source to destination on their network. It is intended by dividing the quantity of data received by ending state finished the quantity package originated from starting point on the network. PDF = $(\mathrm{Pr} / \mathrm{Ps})^{*} 100$

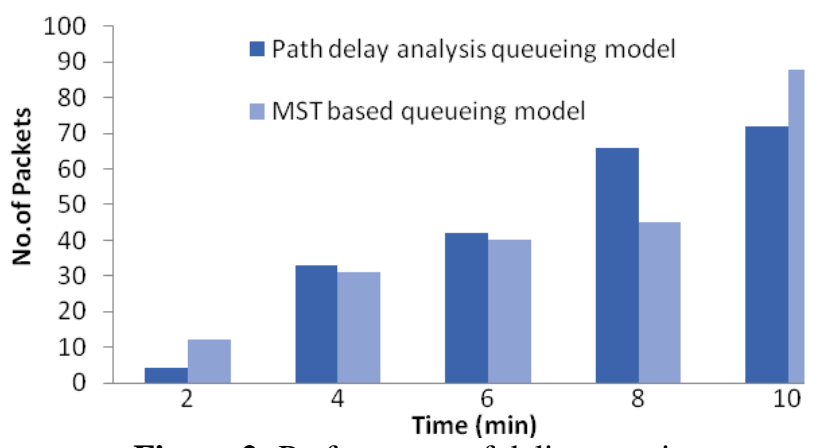

Figure 2: Performance of delivery ratio

Where Pr is total Data established \& Ps is the total data transfer on their network.

\subsection{The End-to-End delay}

They have calculated an average number of delay on the network, it includes all possible delay caused by buffering through route detection latency, queuing at the border queue, retransmission delay on medium access control, spread and move time. That time took a data packet to be crossways a queueing model network from start to ending point on the network.

$\mathrm{D}=(\mathrm{Tr}-\mathrm{Ts})$

Where $\operatorname{Tr}$ receives Time and Ts is sent Time.

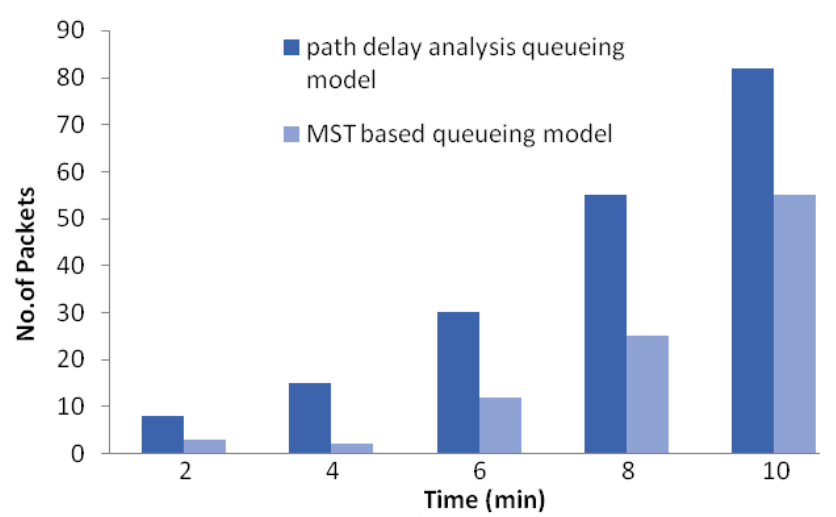

Figure 3: Performance of delay ratio

\section{Conclusion}

The different queueing theory and network channel to minimize the energy consumption based on the FIFO, the MST algorithm is provided. The MST algorithm uses the minimum spanning tree to connect the entire wireless node with the queueing model. However, the system created by the MST algorithm is used to reduce failure node and replace the new FIFO queueing node in the network. We have used low propagation delay network to reduce delay and reduce relay coils in the network. To improve the network performance based on this MST and LPDMR. In our future work to implement the alternative network performance and reduce delay, avoid traffic model on the network.

\section{References}

[1] A. W. Liehr, K. J. Buchenrieder, "Simulating InterProcess Communication with Extended Queueing Networks," Simulation Modelling Practice and Theory, Vol. 18, No. 8, 2015

[2] C. Chiasserini and E. Magli, "Energy Consumption and Image Quality in Wireless Video-Surveillance Networks," September 2002.

[3] I. F. Akyildiz, T. Melodia and K. R. Chowdhury, "A Survey on Wireless Multimedia Sensor Networks," Computer Networks, Vol. 51, No. 14, 2007,

[4] T. Qiu, F. Xia, F. Lin, G. Wu and B. Jin, "Queueing The-ory-Based Path Delay Analysis of Wireless Sensor Net-works," Vol. 11, No. 2, 2011.

[5] T. Qiu, L. Wang, L. Feng and L. Shu, "A New Modeling Method for Vector Processor Pipeline Using Queueing Network," August 2010..

[6] S. A. Khan and S. A. Arshad, "QoS Provisioning Using Hybrid FSO-RF Based Hierarchical Model for Wireless Multimedia Sensor Networks," Vol. 4, No. 1-2, 2009.

[7] R. Andriansyah, T. V. Woensel and F. R. Cruz, "Performance Optimization of Open Zero-Buffer MultiServer Queueing Networks," Vol. 37, No. 8, 2010.

[8] Neelam Srivastava, "Challenges of Next-Generation Wireless Sensor Networks and its impact on Society", volume 1, issue 1, Feb. 2010.

[9] Zhi Sun, Pu Wang, "Border Sense: Border patrol through advanced wireless sensor networks", 2011.

[10] Ian F. Akyildiz, "Signal propagation techniques for wireless underground communication networks", 2009

[11] M. C. K. Wiltshire, "Experimental and theoretical study of magneto-inductive waves supported by onedimensional arrays of "swiss rolls", 15 APRIL 2004.

[12] Zhi Sun and Ian F. Akyildiz, "On Capacity of Magnetic Induction-based Wireless Underground Sensor Networks" 